\title{
The Cape York Meteorites, the Younger Dryas, and Their Possible Association with the Hiawatha and Paterson Impact Craters
}

\author{
Martin Beech ${ }^{1,2}$, Mark Comte ${ }^{2}$, Ian Coulson ${ }^{3}$ \\ ${ }^{1}$ Campion College, the University of Regina, Regina, Canada \\ ${ }^{2}$ Department of Physics, the University of Regina, Regina, Canada \\ ${ }^{3}$ Department of Geology, the University of Regina, Regina, Canada
}

Email address:

beechm@uregina.ca (M. Beech), comte20m@uregina.ca (M. Comte), ian.coulson@uregina.ca (I. Coulson)

\section{To cite this article:}

Martin Beech, Mark Comte, Ian Coulson. The Cape York Meteorites, the Younger Dryas, and Their Possible Association with the Hiawatha and Paterson Impact Craters. American Journal of Astronomy and Astrophysics. Vol. 8, No. 4, 2020, pp. 66-74.

doi: 10.11648/j.ajaa.20200804.11

Received: October 20, 2020; Accepted: November 2, 2020; Published: November 9, 2020

\begin{abstract}
The recent discovery of the Hiawatha and Paterson impact craters in north-western Greenland has motivated three intriguing questions: are they associated with the Cape York meteorites, did they form at the same time, and can one or both of the craters be associated with the abrupt cooling of the Earth, some $10-13,000$ years ago, at the onset of the Younger Dryas. To address the first question, we review the properties of the Cape York meteorites and their associated strewn field. Using the Earth Impact Effects simulator, it is found that the strewn field is generally consistent with the entry of a 2 to 6-m diameter iron asteroid into the Earth's atmosphere some 1 to 2 million years ago. The latter, terrestrial residency age of the meteorites, however, remains preliminary, and further radionuclide analysis is required to confirm the fall epoch. The possibility that the Cape York meteorites are progenitor fragments ejected at the time of crater formation has been investigated with an atmospheric flight program, and while it is possible to account for progenitor fragments traveling the $300-\mathrm{km}$ distance between either crater location and the strewn field, this scenario is deemed unlikely. Indeed, the craters each being in excess of 30-km in diameter would indicate the complete vaporization of the impactors. It is concluded that the Cape York meteorites are unlikely to be related to the formation of either of the craters. Additionally, the $183-\mathrm{km}$ separation between such large craters is remarkable and suggestive of a contemporaneous origin. We investigate this latter possibility, and while it cannot be fully ruled out at the present time, it is, on the basis of Near-Earth Object population statistics, deemed to be highly unlikely that they formed at the same time. This issue, however, will only be fully resolved once improved age estimates become available. Indeed, better crater formation ages will also shed more light upon their possible association with the Younger Dryas onset. With respect to the global climate excursion associated with the Younger Dryas, we review the possibility that the crater progenitor bodies were derived from the Taurid Complex, finding that this scenario is deserving of further study. Moving forwards, however, the conservative hypothesis, that the two craters are temporally distinct, not related to the Cape York meteorites and/or contemporaneous with the Younger Dryas onset, is favored.
\end{abstract}

Keywords: Cape York Meteorites, Impact Craters, The Younger Dryas, The Taurid Complex

\section{Introduction}

The recent discovery of two large, ancient impact craters, with uncertain, but estimated contemporaneous ages, in the Qaanaaq region of north-western Greenland opens-up numerous interesting possibilities and questions concerning the terrestrial impact rate, potential twin-crater production, the origin of the Cape York meteorite strewn field, and the onset of the Younger Dryas - the latter being a distinct cooling period which occurred in the late Pleistocene era some 10 to 13,000 years before present. The newly discovered craters, however, are situated in a still largely 
inaccessible part of the world. Indeed, the remoteness of the high-northern Greenland coast is legendary, and the early expeditionary voyages of John Ross (beginning in 1818) and Robert Peary (from 1886), along with the ill-fated Franklin expedition (starting in 1845) to find the (then fabled, but now realized) North-West Passage have become classic tales of adventure and human endurance under circumstances of extreme duress [1]. North-western Greenland is not entirely un-populated, however, and the Qaanaaq region currently plays host to the Thule Air Base (operational since 1943) which, under the $21^{\text {st }}$ Space Wing of the USAF, is charged with missile defence duties. For all this, while Inuit nations have survived (and historically thrived) in the region for many thousands of years, very little is known about the underlying deep-interior landscape of this area of Greenland $[1,2]$. In the following sections we systematically review the available observations and examine the recently invoked connections that might exist between the craters, the crater progenitors, the Cape York meteorites, the Taurid Complex, and the cosmic micro-spherules located at the Younger Dryas onset boundary.

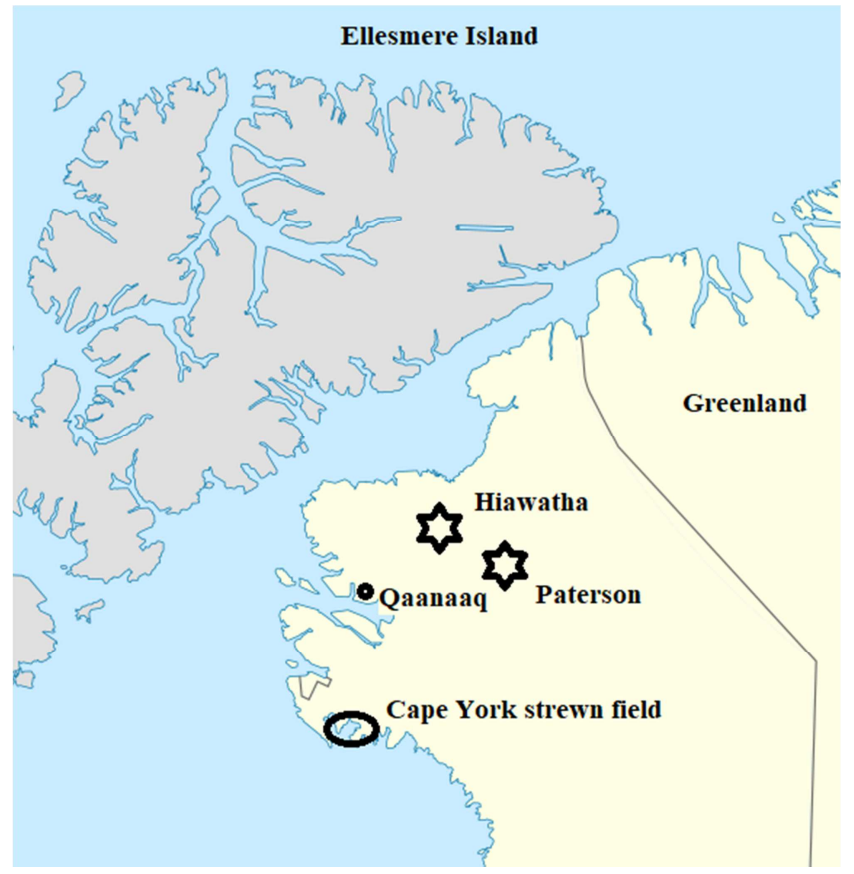

Figure 1. Context map for the locations of the Hiawatha and Paterson craters, along with the Cape York meteorite strewn field. The map scale is approximately 900-km across.

\section{Hiawatha Crater}

The discovery and identification of the Hiawatha impact crater was first reported by Kjær et al. in November 2018 [3] see figure 1 . The crater is some $31 \mathrm{~km}$ in diameter, and while it is partially overlain by ice at the present time, its characteristics (as an impact structure) have been well established with the use of ice-penetrating radar and through mineralogical analysis of water run-off from the Hiawatha Glacier. Indeed, Kjær et al. argue, on the basis of compositions determined for select platinum group elements present within their samples, that the impactor responsible for the Hiawatha crater was a highly fractionated iron asteroid [3]. Additionally, Kjær et al. estimate that the crater formed at some time during the Pleistocene - giving a relatively broad age range of between 2.6 million to 12 thousand years before the present [3].

The materials and presently available data do not conclusively implicate an iron impactor for the origin of the Hiawatha crater, and, as with virtually all large impact structures, there are very few indications as to what kind of solar system object was actually involved [4]. Table 1 is based upon a series of calculations made with a web-based program for determining impact effects [5]. Three compositional variants for the impactor are adopted, ice, stony and nickeliron, and these choices set the density for the impactor as: 1000,3000 and $8000 \mathrm{~kg} / \mathrm{m}^{3}$ respectively. Given that we have no specific knowledge of the angle of the impactor to the local crater horizon, a most probable value of 45 degrees is assumed [6], and the target material is set as crystalline rock appropriate to the Precambrian Greenland basement strata in the area of interest [7]. For each assumed composition and initial velocity, the Earth Impact Effects Program has been used to estimate the size of impactor needed to produce a final crater $31 \mathrm{~km}$ across. For asteroid and short period cometary nuclei a characteristic terrestrial impact speed of between 20 and $25 \mathrm{~km} / \mathrm{s}$ has been adopted. For a long-period cometary nucleus collision the characteristic impact speed is taken as 50 $\mathrm{km} / \mathrm{s}$. In all cases considered the energy associated with the cratering event is of order $3 \times 10^{21}$ Joules, and the typical time interval between impacts capable of producing a crater with the recorded dimensions is of order 5 million years. This latter number is based upon a recurrence time $T_{\mathrm{RE}}$ formula determined by Collins, Melosh and Markus, with $T_{\mathrm{RE}}=109$ $E^{0.78}$, where $E$ (measured in megatons of equivalent TNT explosive energy: $1 \mathrm{Mt}=4.18 \times 10^{15}$ Joules) is the kinetic energy of the impactor at the top of the Earth's atmosphere [5].

Table 1. Impact size estimates for the producing of a crater $31 \mathrm{~km}$ across in a crystalline target. Column 1 is the density of the impactor, column 2 is the impact speed, column 3 indicates the initial impactor size, while column 4 indicates the impactor mass. Calculations are via the Earth Impact Effects Program: https://impact.ese.ic.ac.uk/ImpactEarth/ImpactEffects/.

\begin{tabular}{llll}
\hline Impactor density $\left(\mathbf{k g} / \mathbf{m}^{\mathbf{3}}\right)$ & Impactor speed $(\mathbf{k m} / \mathbf{s})$ & Initial diameter $(\mathbf{k m})$ & Initial $\mathbf{~ m a s s}(\mathbf{k g})$ \\
\hline 1000 (comet) & 20 & 3.4 & $2.06 \times 10^{13}$ \\
1000 & 25 & 3.2 & $5.15 \times 10^{13}$ \\
1000 & 50 & 2.1 & $4.85 \times 10^{12}$ \\
3000 (stony) & 20 & 2.3 & $1.91 \times 10^{13}$ \\
3000 & 25 & 2.0 & $1.26 \times 10^{13}$ \\
8000 (iron) & 20 & 1.5 & $1.41 \times 10^{13}$ \\
8000 & 25 & 1.3 & $9.20 \times 10^{12}$ \\
\hline
\end{tabular}


There are no good statistics, or agreed upon methods for estimating what kind of impactor is most likely to strike the Earth [8]. In general, however, an asteroid having a stony composition is more likely to hit the Earth than a predominantly metallic one, and an asteroid is more likely to hit the Earth than a short period cometary nucleus. Indeed, much less than $1 \%$ of the current Near-Earth Objects are active short period comets [9, 10]. Additionally, it is generally given than an impact with a long period cometary nucleus is much less likely than an impact from a short period cometary nucleus. Sekanina and Yeomans estimate that the Earth is only hit by a kilometer-sized, long period comet once every 43 Million years [11]. On more-firm statistical grounds it is known that about $15 \%$ of Near-Earth asteroids are binary in nature, but, so-far, the observed parent-to-satellite mass ratios are typically high, and only four near-equal mass binary asteroid systems are currently known [12, 13]. Given their respective sizes, a near-equal mass binary would be required if the Hiawatha and Paterson craters were formed under the binary impactor hypothesis. In terms of potential impactor diameter (table 1) the variation between the extremes is about a factor 2.5, but all deduced sizes are well within the realm of known common Near-Earth Object diameters. Accordingly, crater size alone, in this case, tells us essentially nothing about the composition of the impactor.

\section{Paterson Crater}

The discovery and identification of the Paterson impact crater (figure 1) was reported in February 2019 [14, 15]. The name for this crater is adopted upon the suggestion by MacGregor et al., and is given in honor of glaciologist W. S.
B. (Stan) Paterson [14]. This crater is approximately $36 \mathrm{~km}$ in diameter and overlain by some $2 \mathrm{~km}$ of ice, and is located some $183 \mathrm{~km}$ south-east of the Hiawatha crater. In this particular case the identification as an impact structure is based upon the morphology revealed by ice-penetrating radar data. No ice-core sample presently exists for the Paterson crater, and accordingly it may have been produced by a cometary nucleus, a stony or an iron asteroid impact. No reliable estimates are presently available for the crater's formation age.

\section{The Cape York Meteorites}

The existence of iron meteorites in the Cape York region of north-western Greenland was first deduced by Captain James Ross in the early $19^{\text {th }}$ century, but the first actual fragments were found (by non-Inuit explorers, that is) in the late $19^{\text {th }}$ century [16]. A total of 8 (possibly 9) meteorites are now associated with the Cape York strewn field (table 2 and see figure 1), the most recent confirmed specimen (the 250 $\mathrm{kg}$ Tunorput meteorite) being found in 1984 - exactly 100 years after Captain Robert Peary identified the first member, Ahnighito (The Tent), of the strewn field. Indeed, Ahnighito, weighing-in at $30,900 \mathrm{~kg}$ is the $3^{\text {rd }}$ most massive meteorite ever to be discovered - bested only by the Hoba meteorite (Namibia) and the Gancedo fragment of the Campo del Cielo strewn field (Argentina). Many additional members of the Cape York strewn field no-doubt exist. Indeed, fragments broken from Cape York meteorites were extensively traded amongst the Inuit, and artifacts containing Cape York iron have been found at archaeological sites along the entire west coast of Greenland, and well into northern Canada and the north coast of Alaska [1, 2].

Table 2. Members of the Cape York Strewn field. Information from the Meteoritic Bulletin - https://www.lpi.usra.edu/meteor/metbull.php?code=5262, and Buchwald [17]. * This potential transported find, found 25-km west of Qaanaaq (see figure 1) by Casper Nielsen on 15 September 2019, is still provisional, and while identified as an iron meteorite no mineralogical classification and/or direct linkage to the Cape York strewn field has, to date, been published.

\begin{tabular}{lll}
\hline Name & Mass (kg) & Discovered \\
\hline Ahnighhito (The Tent) & 30,880 & 1884 \\
Woman & 3000 & 1897 \\
The Dog & 407 & 1897 \\
Savik I & 3,402 & 1913 \\
Thule & 48.6 & 1955 \\
Savik II & 7.8 & 1961 \\
Agpalilik (The Man) & 20,140 & 1963 \\
Tunorput & 250 & 1984 \\
Thule II* & 31.5 & 2019 \\
\hline
\end{tabular}

The Cape York meteorites are chemically inhomogeneous and belong to the IIIAB group of iron meteorites (that is, they are medium octahedrites containing abundant troilite inclusions). Indeed, the meteorites formed through melt crystallization in an environment affected by a gravitational gradient and within the core of a differentiated asteroid [17, 18]. Measurements of ${ }^{129} \mathrm{Xe}$ and ${ }^{131} \mathrm{Xe}$ in Cape York troilite reveal a cosmic ray exposure (CRE) age of $82 \pm 7 \mathrm{Ma}$, which is much shorter than the typical exposure age of $450 \mathrm{Ma}$ associated with IIIAB irons $[19,20]$. This short exposure age is suggestive of the Cape York meteorites being associated with a relatively recent asteroid break-up event. It is generally taken that the Cape York meteorites fell at least some 10,000 years ago, prior to the arrival of the first Inuit in north-western Greenland [2]. This being said, Nishiizumi et al. have determined terrestrial ages for the Thule and two fragments of what they identify as Cape York (it is not clear which particular main-mass the fragments are from - see table 2) [21]. Specifically, Nishiizumi et al. analyzed the abundance of the cosmic radionuclide ${ }^{36} \mathrm{Cl}$, finding $1.7 \pm 0.3$ 
$\mathrm{dpm} / \mathrm{kg}$ of ${ }^{36} \mathrm{Cl}$ in the Thule meteorite fragment, and $0.44 \pm 0.3$ and $0.76 \pm 0.3 \mathrm{dpm} / \mathrm{kg}$ of ${ }^{36} \mathrm{Cl}$ in the "Cape York" fragments. These measured radionuclide abundances suggest a terrestrial age for the Cape York family of meteorites in the range from 1.1 to 1.7 million years. At face value these terrestrial residency ages indicate that the Cape York meteorites fell during the Calabrian subdivision of the Pleistocene, but it is very much the case that additional analyses of their residency age should be made. There are no distinctive impact structures or plunge pits associated with the find locations of any of the Cape York meteorites discovered to date, and this suggests that the meteorites either fell into a thick icecovered surface, or that any such features were relatively indistinct and have long been eroded and weathered away.

\section{A Five-way Connection}

In terms of possible generic relationships between the Cape York strewn field, and the Hiawatha and Paterson craters, five possible scenarios are logically in play, and these are:

1) The Hiawatha crater, the Paterson crater, and the Cape York meteorite strewn field have no connection to each other, and that they occurred at three distinct and unrelated epochs.

2) The Hiawatha crater and the Paterson crater formed at the same time, but are unrelated to the formation of the Cape York meteorite strewn field.

3) The Hiawatha crater, the Paterson crater, and the Cape York meteorite strewn field were all produced at the same time.

4) The Hiawatha crater is distinct (in age) from the Paterson crater, but related to the Cape York meteorite strewn field.

5) The Paterson crater is distinct (in age) from the Hiawatha crater, but related to the Cape York meteorite strewn field.

None of these scenarios can be fully discarded at present, and crucial to the resolution of the issue will be the determination of good ages, both for the terrestrial ages of the meteorites, and for the individual craters. Our default position is that scenario 1 is the most likely scenario to be true. Firstly, as argued for by MacGregor et al., the probability of two large craters, with centers just $180 \mathrm{~km}$ apart, forming at or close to the same time is extremely low [14]. The twin, near-equal sized, Clearwater craters in Canada, remind us, however, that distinct craters, multiple tens of kilometers in diameter, can appear almost next to each other (just $30 \mathrm{~km}$ apart in the Clearwater case) and yet have very different formation ages (the Clearwater East and West craters have formation ages placed at 460 and 286 million years before present respectively [22]). Second, given that Greenland and specifically the Rae and North Atlantic Cratons and adjacent terrains in the high Arctic, provide for the exposure of some of the oldest rocks on Earth, and that the new crater discoveries are probably a first glimpse of a complex impact history written large upon a very ancient landscape. Indeed, Garde et al. have argued that the eroded remains of what would amount to the oldest and largest impact crater ever found on Earth are exposed within the surface rocks of the North Atlantic Craton, near Maniitsoq, in south-western Greenland [23]. The probability that the Hiawatha and Paterson impact craters were formed simultaneously due to the impact of an equal-mass component, binary asteroid, would additionally seem to be extremely low, but can not, at present, be fully ruled out. The only known, reasonably well constrained with respect to formation age, twin impact structures so far identified on Earth are the Lockne and Målingen craters, in Sweden [24]. In this particular case the craters formed some 460 million years ago, are about $25 \mathrm{~km}$ apart, and have distinctly unequal diameters of 7.5 and 0.7 kilometers respectively.

The low probability that must be associated with the contemporaneous origin for the Hiawatha and Paterson craters make scenarios 2 and 3 highly unlikely. Scenarios 4 and 5 are additionally highly unlikely, varying only in association, due to the low probability that would generally be placed upon a specific impactor being predominantly metallic in nature (i.e., in order to account for the Cape York meteorites). For all this, however, many impact structures are known to be associated with iron meteorite strewn fields - the classic examples being the Campo del Cielo strewn field in Argentina, and the Canyon Diablo iron meteorites associated with the Barringer crater in Arizona [25] - but see below.

If it is true that the Cape York meteorites are associated with one, or possibly both, the Hiawatha and Paterson craters, the question of their large off-set of some $300-\mathrm{km}$ from the impact site (s) demands an explanation. Again, a number of scenarios are possible, or at least can be distinguished.

1. Taking the discovery locations of the Cape York meteorites at face value and assuming little physical transport since they landed, then the distance between the Thule and Ahnighhito finds is in excess of $100 \mathrm{~km}$, and this would make for an exceptionally long (recordbreaking length) strewn field. Additionally, taking the meteorite masses as given, then the direction of flight for the strewn field, which places more massive meteorites down-range, would be from the north-west towards the south-east. While this flight direction is entirely possible, the generation of such a long majoraxis for the strewn field, would require a very shallow angle of entry into the Earth's atmosphere. Shallow entry angles, however, are rare since the probability varies as $d P=2 \cos \eta \sin (\eta) d \eta$, where / is the angle to the horizon - giving $\eta=45$ degrees as the most probable entry angle [6]. Clearly, however, as $\eta \Rightarrow 0$, so the probability of such a trajectory coming about becomes increasingly small.

2. Taking the Thule meteorite to be a transport find from the main Cape York strewn field (by Inuit traders, for example), then the remaining members of the Cape York group present (again if little individual transport has taken place) a near circular strewn field some 25 $\mathrm{km}$ across - this is a more characteristic size for a 
typical meteorite strewn field, and is suggestive of a high angle of entry to the horizon - i.e., consistent with the $\eta=45$ degrees, most likely, value for the entry angle [6]. Support for this scenario can be found by considering a minimum-mass parent body for the known Cape York meteorites. Indeed, table 3 summarizes a set of calculations derived from the Earth Impact Effects Program, with the initial mass set to 58 metric tonnes (the combined mass of the known Cape York meteorites - see table 2), and with the entry angle being set to 45 degrees. The calculations indicate that, for a range of probable initial velocity values, such a minimum mass iron meteoroid, of initial diameter 2.4-m, will begin to fragment at an altitude of about $15-\mathrm{km}$, and suffer catastrophic breakup at an altitude of about $13-\mathrm{km}$ altitude. None of the calculations indicate the formation of any distinctive craters; rather a strewn field with a characteristic size of $L=h$ (fragment) $/ \sin (45) \sim 21 \mathrm{~km}$ is indicated. The results summarized in table 3 change but little if the initial mass is increased to 1000 metric tonnes (corresponding to an initial dimeter of 6.2 meters). In addition, it is noted, that Buchwald indicates that in spite of extensive weathering and surface alteration by human hammering, the Thule, Savik I and Agpalilik meteorites continue to show distinctive regmaglypt features, and these are a clear indicator of atmospheric heating and ablation [17]. It would appear that producing the Cape York strewn field does not pose any specifically awkward problems, and can be explained under the typical scenario of atmospheric fragmentation of a small iron asteroid. For all this, however, the question still remains as to a possible relationship to the Hiawatha and Paterson crater progenitor (s).

Table 3. Minimum mass Cape York impactor model. Column 1 is the assumed initial velocity, while column 2 is a summary of the initial fragmentation altitude, airburst height and strewn field production. Model calculations assume an iron meteorite composition.

\begin{tabular}{ll}
\hline Velocity $(\mathbf{k m} / \mathbf{s})$ & Outcome of atmosphere interaction \\
\hline \multirow{3}{*}{15} & Fragmentation begins at $10 \mathrm{~km}$ altitude \\
& Airburst at $9 \mathrm{~km}$ altitude \\
& Strewn field produced \\
& Fragmentation begins at $16 \mathrm{~km}$ altitude \\
& Airburst at $14 \mathrm{~km}$ altitude \\
& Strewn field produced \\
& Fragmentation begins at $19 \mathrm{~km}$ altitude \\
& Airburst at 17 km altitude \\
& Strewn field produced \\
\hline
\end{tabular}

3. Is it possible that the Cape York meteorites are impactor fragments produced during the excavation of either or both the Hiawatha and the Paterson craters? Under straightforward parabolic flight conditions, the maximum range that can be achieved (following a launch angle of 45 degrees) is given by the formula $R_{\text {max }}=V_{\text {ej }}{ }^{2} / g$, where $V_{\text {ej }}$ is the ejection velocity and $g$ is Earth's surface gravity. Accordingly, for an ejection velocity of $V_{\mathrm{ej}}=1.7 \mathrm{~km} / \mathrm{s}$ a maximum range of order 300 $\mathrm{km}$ from the ejection center could be realized. According to this naïve analysis the Cape York meteorites could in principle be impactor fragments from either the Hiawatha crater or the Paterson crater provide the impactor, of course, was a predominantly iron asteroid. A $2 \mathrm{~km} / \mathrm{s}$ impactor-fragment ejection speed is entirely reasonable in the wake of an initial 20 $\mathrm{km} / \mathrm{s}$ speed for the crater-forming projectile - the maximum ejecta velocity is generally taken as being much smaller and no more than half of the initial impact velocity [26]. This straightforward, order of magnitude, analysis holds true even in the application of a more rigorous numerical integration of the equations of motion including detailed atmospheric drag effects [27]. The key problem with this ejection scenario is that the largest known crater for which iron meteorites (impactor fragments) have survived is that of the $1.2 \mathrm{~km}$ diameter Barringer crater, and no meteorite in this particular case has been found more that $\sim 6 \mathrm{~km}$ away from the crater center (see e.g., Melosh and Collins and the strewn field map that they present therein [26]). Indeed, the key point with respect to craters having sizes comparable to that of the Hiawatha crater $(31 \mathrm{~km})$ and/or the Paterson crater $(36 \mathrm{~km})$ is that the energy involved in the impact $\left(E>10^{21}\right.$ Joules) is so high that the impactor must be completely vaporized. It seems most unlikely, therefore, that the Cape York irons are impactor fragments ejected during the formation of a substantive crater. This being said, Kyte has claimed the discovery of a metal and sulphide-rich carbonaceous chondrite micro-meteorite in a sediment layer of the Pacific Ocean coincident in age (65 million years) with the Chicxulub impact crater [28]. This latter crater has a diameter in excess of $150 \mathrm{~km}$ and is known to be associated with an extensive tektite strewn field. Maier et al. have additionally claimed the detection of chondritic meteorite fragments within a core sample associated with the Moroqweng $(70 \mathrm{~km}$ diameter $)$ impact crater - such fragments presumably being derived from the impactor [29]. In addition, it is entirely possible that impactor fragments could be projected downrange of a crater in the advent of a grazing impact [30]. In this latter highly improbable shallow entry angle situation (recall argument 1) decapitation of the impactor is predicted to occur, but the resultant (primary) impact crater will then be highly elliptical in profile, and this is not the observed situation in either the Hiawatha or Paterson craters. What additionally acts against the impactor-fragment scenario, whether shallow entry angle or not, is the preservation of regmaglypts on (at least) several of the Cape York meteorites. Such distinctive ablation features will not form under the low velocity, impactor-fragment ejecta scenario.

4. While in general a crater produced as a result of a cometary impact would not be expected to yield iron 
meteorite fragments, there is at least the intriguing example set by the Mazapil iron meteorite which fell during the Andromedid meteor storm of November 1885. This particular meteor shower is associated with the now lost and presumed extinct comet 3D/Biela. In this case, the cometary nucleus was observed to undergo a distinct fragmentation event during its 1846 perihelion return, becoming completely lost after its 1852 perihelion passage. Beech has analysed the circumstances of the Mazapil fall, but finds that no clear association can be made between the fall of the meteorite and the circumstances relating to the Andromedid meteor storm [31]. The situation is such that the timing of these two note-worthy events was nothing more than coincidence. In principle, however, cometary nuclei may well accrete (that is, literally absorb and store through impact burying) small iron asteroids / meteoroids as they move through the mainbelt asteroid region, and this, albeit with a very low probability of occurrence, could account for an iron meteorite fall during a cometary meteor shower [32, 33]. The existence of annual or regular meteorite-producing streams has been claimed in the past, but no clear correlation between the fall times of actual meteorites and the activity of any specific annual meteor shower has even been established (although see the discussion relating to the Taurid Complex below) $[34,35]$.

In summary, there appears to be no compelling reason to suppose that the Cape York meteorites must be associated with either the Hiawatha or the Paterson craters. The main concentration of meteorites in the Melville Bay and specifically Meteorite Island of northwest Greenland is entirely consistent with expectations from the entry of a small, no more than 6 to 10 -meter diameter, iron asteroid entering into the Earth's atmosphere some 1 to 2 million years ago. Until good age estimates for the craters can be ascertained, however, the issue of their association (or not) with the Cape York meteorites must remain unresolved.

\section{The Younger Dryas and the Taurid Complex}

An additional twist to the emerging story of the Hiawatha and Paterson craters has recently been introduced by Pino et al. [36]. Specifically, Pino et al, have investigate in great detail the mega-fauna extinctions, and the abrupt climate change anomaly associated with the Younger Dryas. Indeed, drawing upon an inferred similarity of ages, Pino et al. (very) tentatively suggest that the event resulting in the production of the Hiawatha crater might also be responsible for generating the nano-diamonds, micro-spherules, melt-glass, and elevated levels of $\mathrm{Ni}, \mathrm{Co}$ and $\mathrm{C}$ that characterize the (now apparently global [37]) Younger Dryas horizon. This latter association, if real, brings into the already muddied-mix of possibilities an association with the Taurid Complex. Long a system of great debate, the Taurid Complex is generally taken to be composed of the debris derived from the break-up of a large 50-km sized comet (possibly Kuiper belt object) some 20 - 30,000 years before present [38-40]. Amongst the recognized components of the Taurid Complex are the 5-km sized comet $2 \mathrm{P} /$ Encke (unique in being a short period comet dynamically decoupled from the influence of Jupiter [41]), and the multi-kilometer sized near-Earth asteroids (NEA's) 2201 Oljato (often touted as a potential transitional comet/asteroid object), 4341 Poseidon, 306367 Nut, 4197 Morpheus and possibly 2212 Hephaistos [42, 43]. Additional Taurid Complex members are the NEA 4183 Cuno (a rare Qtype asteroid with spectral characteristics akin to L and LL ordinary chondrites) and possibly the 2-km sized 4486 Mithra which is suspected of being a contact binary. The Taurid Complex also contains the meteoroid streams associated with the Northern and Southern Taurids (which are dynamically linked to comet $2 \mathrm{P} /$ Encke) as well as the meteoroid streams associated with day-time beta-Taurids and zeta-Perseid meteor showers (dynamically related to asteroid 2004 TG10). Furthermore, a component of the Taurid meteoroid stream is known to be in a 7:2 mean motion resonance with Jupiter, and this not only restricts the dispersal of the meteoroids within the stream, but it also controls the appearance of distinctive fireball outburst events $[42,44,45]$ - these being times at which an enhanced flux of particularly large meteoroids encounter the Earth. The betaTaurid stream is particularly interesting with respect to the topic under discussion here since it has been identified as the parent stream to the Tunguska impactor [38, 46, 47]. This latter event being associated with the catastrophic airburst of a 50-meter sized cometary fragment over Siberia on June 30, 1908 [48]). Additionally, the Farmington meteorite (L5 chondrite), which fell on 25 June 1890 (and which has the shortest known cosmic-ray exposure age of any L-chondrite meteorite - just 25,000 years), the Tagish Lake meteorite (ungrouped C2) which fell in 2000, the Maribo meteorite (CM2 chondrite) which fell in 2009, and the Sutter's Mill meteorite (CM2 chondrite) which fell in 2012, have all been identified as potential Taurid Complex members [46, 49]. Given the characteristic encounter speeds of Taurid stream components, $27 \mathrm{~km} / \mathrm{s}$ for the Southern Taurids and $29 \mathrm{~km} / \mathrm{s}$ for the northern Taurids, any one of the crater formation scenarios outlined in table 1 could be accommodated for by an appropriately-sized Taurid Complex asteroid/comet impactor. Likewise, given that asteroid 4486 Mithra is a contact binary, and that the break-up of the parent object to the Taurid Complex was gentle enough to form similar such asteroid pairs, then this opens-up the possibility for a simultaneous origin of the Hiawatha and Paterson craters - if, of course, they actually have an origin that can be associated with the Taurid Complex.

\section{Discussion}

It has been written many times before now that one can speculate endlessly in the absence of evidence, and 
apparently meaningful connections, where no real connections exist, can be easily forged from fragmentary data. Such absence of detailed data and epoch specific evidence, however, should not act to stifle reasonable debate and scenario construction. Indeed, observation and experiment can only be interpreted through pre-existing theories and convictions. At this stage, however, it would seem that the entire debate concerning the potential connection between the Hiawatha crater, the Paterson crater, the Cape York meteorite strewn field, the Younger Dryas onset and the Taurid Complex hinges upon the determination of accurate ages. That is, what is required moving forward, is a much better understanding of when the various Greenland craters formed, a better understanding of the chemical make-up of the crater producing impactors, and a much better understanding of the disintegration history of the Taurid Complex parent body especially with respect to the timing sequence of break-up events, and the mass distribution of components produced during fragmentation events. Along these latter lines, Napier has argued that the cooling associated with the onset of the Younger Dryas might have come about through the Earth encountering an extensive dust and debris cloud derived through the successive sublimation and fragmentation of a kilometer sized, Taurid Complex, cometary nucleus [50]. While this model potentially accounts for the onset of the Younger Dryas, along with the nanodiamonds and melt-glass spherules found at the Younger Dryas boundary, it does not invoke an association with a large cratering event [36]. Indeed, as noted by Napier, there is a timing issue with respect to the a priori expectation of finding a large crater, such as the Hiawatha and Paterson craters, with an age of order 13,000 years [49]. If such cratering events do occur on a repeat timescale of some 5 million years (recall section 2), then the expectation of there being a crater associated with the onset of the Younger Dryas is about 1 in 400 . This argument once again underscores the great importance of narrowing down the formation ages for the Hiawatha and Paterson craters. Some progress in this area has recently been achieved by Garde et al. who have investigated the degradation of organic matter within the impactite grains sampled from the outwash from the Hiawatha crater floor [51]. Accordingly, they find a maximum crater formation age of between 3 to 2.4 million years before present. This result is still consistent with the crater being associated with the Cape York meteorites, and it also indicates that the Hiawatha crater is the youngest known, large impact crater on Earth.

The data supporting a strong climatic perturbation during the Younger Dryas now seems unassailable, and an impact event of the magnitude associated with the formation of a 30 $40 \mathrm{~km}$ diameter crater could conceivably be the trigger mechanism [36, 51] - although alternative nonextraterrestrial-driven scenarios, such as the inhibition of the ocean's thermohaline circulation current, have also been presented [52]). This latter mechanism, however, does not explain the presence of the melt-glass and micro-meteorites at the Younger Dryas horizon recently identified by Pino et al.
[36]. Finally, it should be noted, that the current age estimates placed upon the occurrence of the Younger Dryas (10 - 13,000 years before the present) and the terrestrial residency age of the Cape York meteorites (of order 1.5 million years before present) suggest that if the Hiawatha and/or Paterson craters are linked to the Cape York meteorites, then they cannot account for the onset of the Younger Dryas and visa versa.

\section{Conclusions}

In the absence of any well constrained crater age estimates, scenario 1 , as outlined above, in which there is no established causal relationship between the formation of the Hiawatha crater, the Paterson crater, and the Cape York meteorite strewn field, must be the default option moving forwards. Any association between the formation epoch of the Hiawatha crater and the onset of the Younger Dryas must also be taken as unproven at the present time. Such conclusions, however, must also be seen as provisional provisional, that is, upon the determination of better constraints on the crater formation ages. Establishing a higher likelihood for any one of the five scenarios discussed above, as well as strengthening and/or discounting potential connections to the Taurid Complex and the Younger Dryas, will be a challenge, but challenge is exactly what science and understanding is all about.

\section{References}

[1] P. A. M. Huntington 2002. Robert E. Peary and the Cape York meteorites. Polar Geophysics, 26, 53-65.

[2] M. Appelt, et al. 2015. The cultural history of the Innaanganeq / Cape York Meteorites.

https://www.forskningsdatabasen.dk/en/catalog/2393846340.

[3] K. H. Kjær et al. 2018. A large impact crater beneath Hiawatha Glacier in northwest Greenland. Science Advancement, 4, eaar8173.

[4] D. W. Hughes 2002. A comparison between terrestrial, Cytherean and lunar impact cratering records. Monthly Notices of the Royal Astronomical Society, 334, 713-720.

[5] G. S. Collins, H. J. Melosh and R. A. Markus 2005. Earth Impact Effects Program: A Web-based computer program for calculating the regional environmental consequences of a meteoroid impact on Earth. Meteoritics and Planetary Sciences, 40, 817-840.

[6] D. W. Hughes 1993. Meteorite incidence angles. Journal of the British Astronomical Association, 103, 123-126.

[7] A. P. Nutman, P. R. Dawes, F. Kalsbeek, F. and M. A. Hamilton 2008. Palaeoproterozoic and Archaean gneiss complexes in northern Greenland: Palaeoproterozoic terrane assembly in the High Arctic. Precambrian Research, 161, 419-451.

[8] W. M. Napier 2004. A mechanism for interstellar panspermia. Monthly Notices of the Royal Astronomical Society, 348, 4651 . 
[9] M. Boslough 2013. Greenland Pt anomaly may point to noncataclysmic Cape York meteorite entry. Proceedings of the National Academy of Sciences, 110, E5035.

[10] D. A. Yeomans 2013. Near-Earth Objects. Princeton University Press, Princeton.

[11] Z. Sekanina and D. H. Yeomans 1984. Close encounters and collisions of comets with the Earth. Astronomical Journal, 89, 154-161.

[12] K. J. Walsh and S. A. Jacobson 2015. In, Asteroids IV, P. Michel et al. (eds.), University of Arizona Press, Tucson. pp: 375-393.

[13] P. A. Taylor, et al. 2019. Radar and optical observations of equal-mass binary near-Earth asteroids (190166) 2005 UP156 and 2017 YE5. 50th Lunar and Planetary Science Conference 2019 (LPI Contrib. No. 2132).

[14] J. A. MacGegor et al. 2019. A Possible Second Large Subglacial Impact Crater in Northwest Greenland. Geophysical Research letters, 46, 1496-1504.

[15] J. Klokočník et al. 2020. Support for two subglacial impact craters in nortwest Greenland from Earth gravity model EIGEN 6C4 and other data. Tectonophysics. https/doe.org/10.1016/j.tecto.2020.228396.

[16] R. E. Peary 1898. Northward over the ice, volume II, Frederick A. Stokes Company, New York.

[17] V. F. Buchwald 1975. Handbook of Iron Meteorites, Volume 2. University of California Press.

[18] A. Kracher, G. Kurat and V. F. Buchwald 1977. Cape York: The extraordinary meteorite and its implication mineralogy of an ordinary iron for the genesis of III AB irons. Geochemical Journal, 11, 207-217.

[19] K. J. Mathew and K. Marti 2009. Galactic cosmic rayproduced 129Xe and 131Xe excesses in troilites of the Cape York iron meteorite. Meteoritics and Planetary Sciences, 44, 107-114.

[20] O. Eugster, G. F. Herzog, K. Marti, and M. W. Caffee 2006. In Meteorites and the Early Solar System II. D. S Lauretta \& H. Y. McSween (Eds). University of Arizona Press, Tucson. pp. 829-851.

[21] K. Nishiizumi et al. 1987. Terrestrial ages of Antarctic and Greenland meteorites. Meteoritics and Planetary Sciences, 22, 473 .

[22] M. Schmieder et al. 2015. New 40Ar/39Ar dating of the Clearwater Lake impact structures (Quebec, Canada) - not the binary asteroid impact it seems? Geochimica et Cosmochimica Acta, 148, 304-324.

[23] A. A. Garde, I. McDonald, B. Dyck and N. Keulen 2012. Searching for giant, ancient impact structures on Earth: The Mesoarchaean Maniitsoq structure, West Greenland. Earth and Planetary Science Letters, 197, 337-338.

[24] J. Ormö, E., Sturkell, C., Alwmark, and H. J. Melosh 2014. First known terrestrial impact of a binary asteroid from a main belt breakup event. Nature Scientific Reports, 4: 6724.

[25] H. J. Melosh and G. S. Collins 2005. Meteor crater formed by low-velocity impact. Nature, 434, 157.
[26] H. J. Melosh 1985. Impact cratering mechanics: relationship between the shock wave and excavation flow. Icarus, 62, 339343.

[27] M. Beech, M. Comte, and I. M. Coulson 2019. The Production of Terrestrial Meteorites - Moon accretion and lithopanspermia. American Journal of Astronomy and Astrophysics, 7, 1-9.

[28] F. T. Kyte 1998. A meteorite from the Cretaceous/Tertiary boundary. Nature, 396, 237-239.

[29] W. D. Maier et al. 2006. Discovery of a 25-cm asteroid clast in the giant Morokweng impact crater, South Africa. Nature, 441, 203-206.

[30] M. Beech 2014. Grazing Impacts Upon Earth's Surface: Towards an Understanding of the Rio Cuarto Crater Field. Earth, Moon, and Planets, 113, 53-71.

[31] M. Beech 2002. The Mazapil meteorite: from paradigm to periphery. Meteoritics and Planetary Sciences, 37, 649-660.

[32] M. J. Cintala 1981. Meteoroid impact into short-period comet nuclei. Nature, 291, 134-136.

[33] M. Beech and K. Gauer 2002. Cosmic roulette: comets in the main belt asteroid region. Earth, Moon and Planets, 88, 211221.

[34] M. Beech 2006. Canadian fireball activity from 1962 to 1989. WGN, the Journal of IMO, 34: 4, 104-110.

[35] A. Pauls, and B. Gladman 2010. Decoherence time scales for "meteoroid streams". Meteoritics and Planetary Sciences, 40, $1241-1256$.

[36] M. Pino et al. 2019. Sedimentary record from Patagonia, southern Chile supports cosmic-impact triggering of biomass burning, climate change, and megafaunal extinctions at $12.8 \mathrm{ka}$ Nature Science Reports, 9, Article number: 4413.

[37] A. Moore et al. 2020. Evidence of cosmic impact at Abu Hureyra, Syria at the Younger Dryas onset $(\sim 12.8 \mathrm{ka})$ : high temperature melting at $>2200^{\circ}$ C. Nature Scientific Reports, 10: 4185 .

[38] V. Clube and W. M. Napier 1990. The Cosmic Winter, Blackwell, Oxford.

[39] D. I. Steel and D. D. Asher 1996. The orbital dispersion of the macroscopic Taurid objects. Monthly Notices of the Royal Astronomical Society, 280, 806-822.

[40] W. M. Napier 2010. Paleolithic extinctions and the Taurid complex. Monthly Notices of the Royal Astronomical Society, 405, 1901-1906.

[41] Levison et al. 2006. On the origin of the unusual orbit of 2P/Encke. Icarus, 182, 161-168.

[42] D. Asher, and V. Clube 1993. An extraterrestrial influence during the current glacial-interglacial. Quarterly Journal of the Royal Astronomical Society, 34, 481-511.

[43] P. B. Babadzhanov 2001. Search for meteor showers associated with near-Earth asteroids: I. Taurid Complex. Astronomy and Astrophysics, 373, 329-335.

[44] M. Beech, M. Hargrove, and P. Brown 2004. The running of the bulls: a review of Taurid fireball activity since 1962. The Observatory, 124, 277-284. 
[45] D. Clark, P. Wiegert and P. G. Brown 2019. The 2019 Taurid resonant swarm: prospects for ground detection of small NEOs. Monthly Notices of the Royal Astronomical Society, 487, L35-L39.

[46] L. Kresak 1978. The Tunguska object: a fragment of comet Encke? Bulletin of the Astronomical Institute of Czechoslovakia, 29, 129-134.

[47] D. D. Asher, and D. I. Steel 1998. On the possible relation between the Tunguska bolide and comet Encke. Planetary and Space Science, 46, 205-211.

[48] L. Foschini et al. 2019. The atmospheric fragmentation of the 1908 Tunguska Cosmic Body: reconsidering the possibility of a ground impact. https://arxiv.org/abs/1810.07427
[49] C. Tubiana, et al. 2015. P/Encke, the Taurid complex NEOs and the Maribo and Sutter's Mill meteorites. Astronomy and Astrophysics, 584, A97.

[50] W. M. Napier 2019. The hazard from fragmenting comets. Monthly Notices of the Royal Astronomical Society, 488, 1822-1827.

[51] A. Garde et al. 2020. Pleistocene organic matter modified by the Hiawatha impact, northwest Greenland. Geology, 48, 867-871.

[52] W. S. Broecker 2006. Was the Younger Dryas triggered by a flood? Science, 312, 1146-1148. 